\title{
Statistical Test of Expression Pattern (STEPath): a new strategy to integrate gene expression data with genomic information in individual and meta-analysis studies
}

Paolo Martini ${ }^{1,2}$, Davide Risso ${ }^{3}$, Gabriele Sales ${ }^{3}$, Chiara Romualdi ${ }^{2}$, Gerolamo Lanfranchi ${ }^{1,2^{*}}$ and Stefano Cagnin ${ }^{1,2^{*}}$

\begin{abstract}
Background: In the last decades, microarray technology has spread, leading to a dramatic increase of publicly available datasets. The first statistical tools developed were focused on the identification of significant differentially expressed genes. Later, researchers moved toward the systematic integration of gene expression profiles with additional biological information, such as chromosomal location, ontological annotations or sequence features. The analysis of gene expression linked to physical location of genes on chromosomes allows the identification of transcriptionally imbalanced regions, while, Gene Set Analysis focuses on the detection of coordinated changes in transcriptional levels among sets of biologically related genes.

In this field, meta-analysis offers the possibility to compare different studies, addressing the same biological question to fully exploit public gene expression datasets.

Results: We describe STEPath, a method that starts from gene expression profiles and integrates the analysis of imbalanced region as an a priori step before performing gene set analysis. The application of STEPath in individual studies produced gene set scores weighted by chromosomal activation. As a final step, we propose a way to compare these scores across different studies (meta-analysis) on related biological issues. One complication with meta-analysis is batch effects, which occur because molecular measurements are affected by laboratory conditions, reagent lots and personnel differences. Major problems occur when batch effects are correlated with an outcome of interest and lead to incorrect conclusions. We evaluated the power of combining chromosome mapping and gene set enrichment analysis, performing the analysis on a dataset of leukaemia (example of individual study) and on a dataset of skeletal muscle diseases (meta-analysis approach).

In leukaemia, we identified the Hox gene set, a gene set closely related to the pathology that other algorithms of gene set analysis do not identify, while the meta-analysis approach on muscular disease discriminates between related pathologies and correlates similar ones from different studies.
\end{abstract}

Conclusions: STEPath is a new method that integrates gene expression profiles, genomic co-expressed regions and the information about the biological function of genes. The usage of the STEPath-computed gene set scores overcomes batch effects in the meta-analysis approaches allowing the direct comparison of different pathologies and different studies on a gene set activation level.

\footnotetext{
* Correspondence: gerolamo.lanfranchi@unipd.it; stefanoc@cribi.unipd.it

'CRIBI Biotechnology Centre, University of Padova, via U. Bassi 58/B, 35121

Padova, Italy

Full list of author information is available at the end of the article
} 


\section{Background}

In the last decades, microarray technology has seen such an explosion of applications as to become a standard tool in biomedical research. It has allowed the discovery of many prognostic genome markers related to the development of pathologies [1-6]. The spreading process has brought a dramatic increase in the number of publicly available datasets [7-9].

Given the high-throughput nature of microarrays, statistical and bioinformatic methods were required to analyse such large amounts of data. Initial studies were focused on the identification of differentially expressed genes and their significance in many experimental designs (gene by gene approach). This analysis is timeconsuming and sometimes ineffective because derived gene lists have to be interpreted, searching for patterns of genes that have similar function or are involved in particular processes [10]. This approach revealed that genes that are identified as differentially expressed often do not correlate with the phenotype under investigation. Furthermore, their consistency often decreases when different studies on the same biological issue are compared (meta-analysis approach) [11].

Meta-analysis may be broadly defined as the quantitative review and synthesis of the results of related but independent studies [12]. Different groups demonstrated its applicability to microarray data. Rhodes [13] applied metaanalysis to combine four datasets on prostate cancer to determine genes that are differentially expressed between clinically localized prostate and benign tissue. Parmigiani [14] performed a cross-study comparison of gene expression for the molecular classification of lung cancer. Park and Stegall [15] combined publicly available datasets and their own microarray datasets to investigate the detection of cytokine gene expression in human kidney. Meta-analysis studies clearly showed that the different lists of differentially expressed genes from different studies overlap poorly due to the complicated experimental variables embedded in array experiments. This suggests that a pathway/gene set-based approach could improve the performance of this type of comparison [16].

To improve microarray data analysis, the first tools developed were based on the integration of external genomic information such as gene location [17-19], ontological annotations [20-23] or sequence features [24].

Several methods were devised to analyse gene expression as a function of physical location of genes on chromosomes. These approaches, collectively referred to as "chromosome mapping", were applied to microarray data of cancer studies. The studies identified regions with transcriptional imbalances that reflected large chromosomal aberrations typical of such pathologies. Examples of these applications are the Locally Adaptive statistical
Procedure (LAP) [17] and the MicroArray Chromosome Analysis Tool (MACAT) [18]. LAP was applied to compare gene expression data of acute myeloid leukaemia (AML) with and without trisomy on chromosome 8. LAP correctly identified the over-expressed region on chromosome 8 of patients where DNA amplification was present. MACAT was applied to compare T and B lymphocytes from patients with acute lymphoblastic leukaemia (ALL), identifying a marked over-expression of the region that contains genes of class II major histocompatibility complex (MHCII, chr:6p21.33-6p21.2) in the B lineage. Recently, a chromosome mapping approach based on the Haar Wavelet transformation (Chromowave) [19] was applied to a dataset of Huntington's disease. The study demonstrated that the aberrant interaction between the mutant huntingtin protein and its transcriptional co-activators, such as histone acetyltransferase, leads to large areas of transcriptional imbalances [25].

A more popular method for the integration of gene expression profiles with additional information is based on ontological and pathway annotations and is called Gene Set Analysis (GSA). This approach evaluates gene expression profiles among groups of related genes (gene sets), seeking coordinated changes in the expression levels of subsets of gene members. Usually, GSA has three main steps: a) computing associations of each expression pattern with a phenotype; b) computing enrichment scores for analysed gene sets; c) computing the global p-value and q-value for every tested gene set based on the appropriate permutation test. Several implementations of the GSA approach are now available, such as the algorithms developed by Subramanian (Gene Set Enrichment Analysis; GSEA) [22], Tian (sigPathway) [23], Efron (with the improvement based on the use of the maxmean statistic for summarizing gene sets) [26] and Goeman with Global Test [21].

Recently, Szabó [27] combined mRNA and comparative genome hybridization results, revealing that the major pathogenetic pathways involved in adrenocortical tumours are related to regions with aberrant gene expression. This work is an example of how the integration of different genomic information is useful to gain new insight into a pathology by exploiting available datasets. We believe that an important shortcoming of Szabó's described method is that it is based only on differentially expressed genes thus defining a strict cut-off without considering the actual level of expression.

Here, we propose a new procedure, STEPath (Statistical Test of Expression Pattern), that scores and integrates chromosomal region activation as an a priori step before performing GSA. The result of this analysis is a global expression value of gene sets weighted by chromosomal 
region activation. The plasticity of the chromosome architecture was recently debated due to the identification of transcription factories [28,29], but there are no bioinformatic algorithms that consider this aspect in the gene set analysis. Three public datasets were tested. We demonstrated that the combination of gene expression profiles, chromosome mapping and gene set analysis produced gene set scores suitable to compare different studies in a meta-analysis approach.

\section{Results and Discussion STEPath Algorithm}

We implemented a new gene expression analysis method that takes into account $i$ ) the activation or repression of genes in chromosome regions [30] and ii) the evidence that intensive transcription at one locus frequently spills over in physically adjacent loci [31]. The STEPath algorithm allows scoring and integrating these aspects of gene regulation (i and ii; Step 1) before performing gene set analysis (Step 2). Gene set scores from step 2 can be used for meta-analysis studies (Step 3).

\section{Step 1}

To integrate physical locations of genes, STEPath measures the association of a gene expression profile with a phenotype (e.g., Significance Analysis of Microarrays (SAM) statistics [32]), rescaling it on the expression levels of the neighbour genes. We analysed each gene in relation to the y closest up- and down-regulated genes. Using a permutational approach, we tested the following hypothesis: $H^{O}$, the region did not show differential expression; $H^{1}$, the region is differentially expressed.

\section{Step 2}

After the computation of chromosome profiles using significant regions, STEPath performs a gene set analysis using SAM statistics [32] smoothed according to the chromosome profiles. We defined this step as a gentle integration of the chromosome profile because the smoothing process does not penalize gene scores; instead, it attributes to gene members of differentially expressed regions an additional score proportional to their own SAM score and to the local profile. This method enhances particular signals along chromosomes that are buried in the background due either to sample or technical heterogeneity that could profoundly affect microarray reproducibility.

Using the smoothed statistic, an up- and down-regulation value for every gene set was calculated. As GSA relies on the quality of annotation and dimension of gene sets, it is possible that the signal of a small group of coordinated genes becomes lost in non-specific signals. If this could be an analysis limitation, we increased GSA power by adopting two approaches: a) we analysed the most-used database repositories for gene sets, and b) we extracted portions of pathways showing coordinated expression. a) We have compiled gene sets from Gene Ontology (GO) [33], Kyoto Encyclopedia of Genes and Genomes (KEGG) [33-36], BioCyc [37], BioCarta [38], SuperArray [39] and WikiPathways [40]. The resulting database includes Cellular Component, Molecular Function and Biological Process (GO derived) sets, manually curated functional pathways from BioCarta, metabolic pathways from KEGG and specific pathways for quantitative Real Time PCR (qRT-PCR) and microarray experiments from SuperArray. If different annotations of the same biological aspect may produce redundancies, they also retain specific differences and provide both robustness and specificity when correlated simultaneously to a phenotype (see Additional file 1; Figure S1).

b) According to Efron [26] and later confirmed by Song [41], splitting up and down portions of gene sets improves the statistical power of approaches where the mean of a statistic is used to score gene sets.

Up- and down-regulation scores were independently tested for significance using a gene-based permutation approach. We tested the null hypothesis, $H^{0}$, that the gene set shows the same pattern of association with the phenotype compared to the rest of the genes. The q-values were computed using the Benjamini Hochberg algorithm [42].

\section{Step 3}

In meta-analysis studies, step 3 primarily aims to determine if the results from one study are confirmed in other independent studies.

For an individual study, the STEPath procedure (Step 1 plus Step 2) produces a list of gene sets with summarization values (Gup and Gdown, see Methods) and an associated q-value. Different pathologies can then be directly compared using the gene set summarization values produced for individual studies.

\section{Individual analysis of Leukaemia Dataset}

We tested STEPath on an expression profile dataset of patients affected by Acute Lymphoblastic Leukaemia (ALL; 16 with and 90 without translocation of the Mixed-lineage leukaemia (MLL) gene).

Raw expression data (CEL files) were downloaded from the GEO database (GEO series ID: GSE14062), processed using a gene-based custom Chip Definition File (CDF) [43] to better define the chip [44], and normalized using the Robust Multichip Average (RMA) expression summary [45]. We recovered expression values for 15,953 genes. Using STEPath, we directly compared ALL with (ALL/MLL+) and without MLL translocation (ALL/ MLL-), seeking evidence specific for MLL translocation. Genes without chromosomal location information and genes on chromosome Y were filtered out because paucity of gene expression data precludes the application of chromosome mapping. 
This dataset was used to analyse the performance of the main modules implemented in STEPath: chromosome mapping (Step 1) and gene set analysis (Step 2).

\section{Step 1}

Using our implementation, we were able to identify a spectrum of possible imbalanced regions across all chromosomes (see Additional file 1; Figure S2). We identified the down-regulation of the region that contains the MLL gene (Figure 1A; Additional file 2; Table S1). MLL is characterized by a chromosome rearrangement, disrupting its correct localization and transcriptional regulation [46].

Our method highlights interesting imbalanced regions that contain genes involved in ALL pathology: 1) the region containing the Homeobox genes (HOX cluster) on chromosome 7 (Figure 1B; Additional file 2; Table S2), and 2) the region containing Meis homeobox 1 (MEIS1) on chromosome 2 (Figure 1C; Additional file 2; Table S3).

As discussed by Zangrando [47], HOX genes and MEIS1 are up-regulated in ALL, representing a discriminant signature that separates ALL/MLL+ from ALL/ MLL-. Another gene involved in the discrimination between ALL/MLL+ and ALL/MLL- patients is the chondroitin sulfate proteoglycan 4 (CSPG4/NG2). NG2 encodes for a transmembrane protein located in the leukaemic cell membrane and proposed as a marker for rapid classification of ALL with MLL translocation [48]. Zangrando [47] used SAM and Predicted Analysis of Microarray (PAM) [49] to identify this discriminant gene. Our method pointed out this gene as well, evidencing the goodness of our algorithm (Figure 1D; Additional file 2; Table S4).

We compared our results with the most used approaches to detect imbalanced regions, namely LAP [17] and MACAT [18]. We ran these algorithms using the suggested number of permutations (10,000 for LAP and 1,000 for MACAT, see Additional file 1; Figure S3 and S4) and also with our settings (100 permutations, see Additional file 1; Figure S5 and S6). Different numbers of permutations did not result in relevant differences in the detected regions.

The comparison between LAP results (see Additional file 1; Figure S3) and STEPath chromosome mapping (see Additional file 1; Figure S2) shows that our approach identifies more regions than LAP. We decided to preserve information since this is not an independent procedure, but it is later piped into a gene set analysis. Even if LAP identifies a smaller number of imbalanced regions, these span larger chromosome portions, such as those on chromosome 3 (see Additional file 1; Figure S3), causing difficult interpretation of the results.

In contrast with LAP, our procedure limits imbalanced regions to small portions of interest that are easier to visualize and relate to the studied phenotype. Indeed, the LAP procedure fails to identify as imbalanced the
MLL region on chromosome 11 and the $\mathrm{HOX}$ genes cluster on chromosome 7 (Figure 2 and Additional file 1; Figure S3). LAP identified MEIS1 region on chromosome 2, but this region spans about $45 \mathrm{Mbp}$ (from $\sim 30 \mathrm{Mbp}$ to $\sim 75 \mathrm{Mbp}$, Figure 2 and Additional file 1; Figure S3). Our algorithm reduces the region to 11 Mbp (from $62 \mathrm{Mbp}$ to $73 \mathrm{Mbp}$ ), focusing on truly disease-related genes. These results demonstrate that our algorithm seems to be more sensitive than LAP in the identification of important imbalanced regions involved in ALL.

Furthermore, our procedure reaches a greater sensitivity than MACAT in the detection of regions on chromosome 2, 7, 11 and 15 (Figure 2). The profile of chromosome 11 produced with MACAT (see Additional file 1, Figure S4) is greatly perturbed, and it is difficult to identify interesting regions. MACAT also failed to evidence the MLL region (Figure 2 and Additional file 1, Figure S4).

\section{Step 2}

We completed the STEPath procedure by integrating chromosomal profile information in the module that performs gene set analysis.

The choice between gene (e.g., GSEA implemented in the limma $R$ package [22] and sigPathway [23]) and sample permutations (e.g., Global test [21], Principal Coordinates and Hotelling's $\mathrm{T}^{2}$ (PCOT2) [50] and Significance Analysis of Function and Expression (SAFE) [20]) has been an object of debate in the literature, as demonstrated by Efron's [26] and Goeman's [10] papers. After evaluating the limits and peculiarities of both permutational approaches, we chose the gene permutation model that seems to better fit our null hypothesis (as it is stated in step 2 - b of the algorithm description). We compared results obtained from STEPath with two other implementations of GSA both based on gene label permutations: GSEA implemented in the limma $\mathrm{R}$ package [22] and sigPathway [23]. STEPath is the only procedure that can identify the activated HOX gene set (Table 1). Dysregulation of HOX gene family members was found to be a dominant mechanism of leukaemic transformation induced by chimeric MLL oncogenes [51,52].

To evaluate the contribution of chromosome profile information, we performed our GSA procedure (STEPath) with and without chromosome profile correction. In both cases, STEPath was able to identify the HOX gene set probably due to a separate evaluation of up- and down-regulated genes. However, the integration of gene location with gene set analysis allows the combination of different levels of biological information (co-expressed/ regulated genes) and helps to correctly identify diseaserelated genes, since they have a different position in a rank evaluation (Table 1). To confirm this effect, we also 


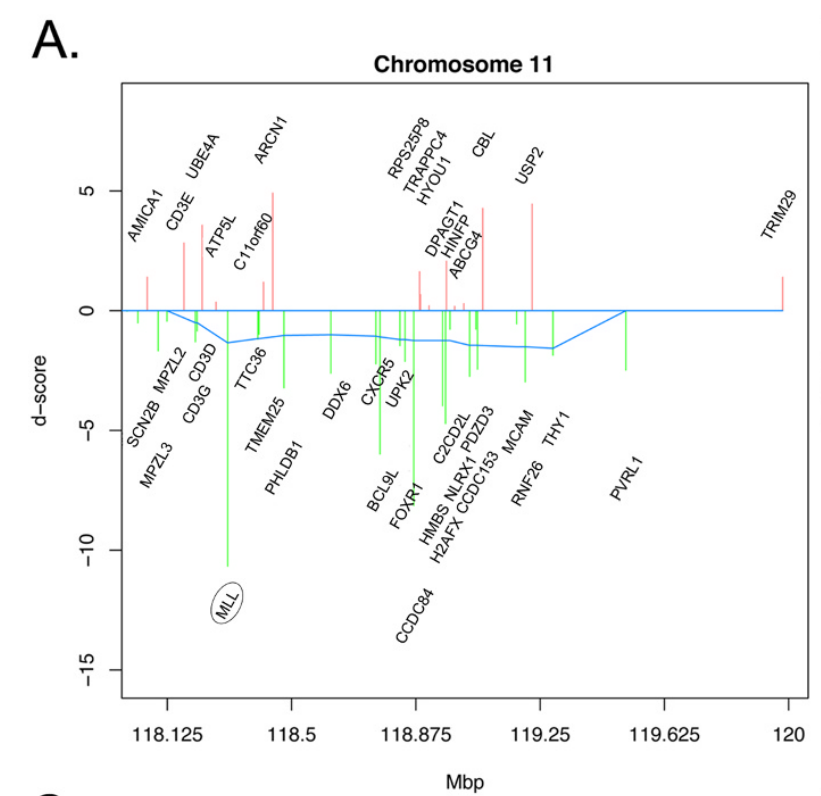

C.

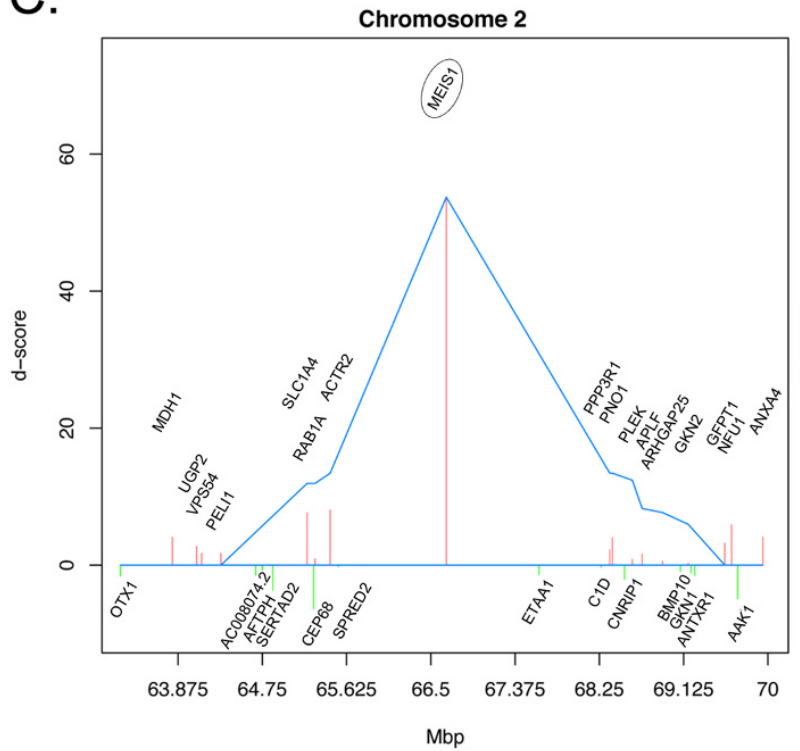

B.

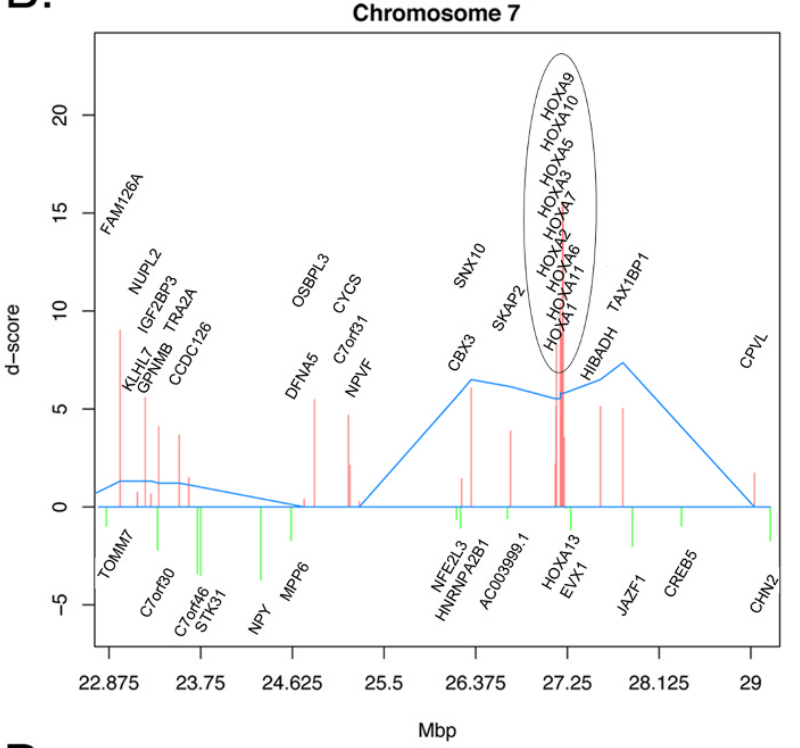

D.

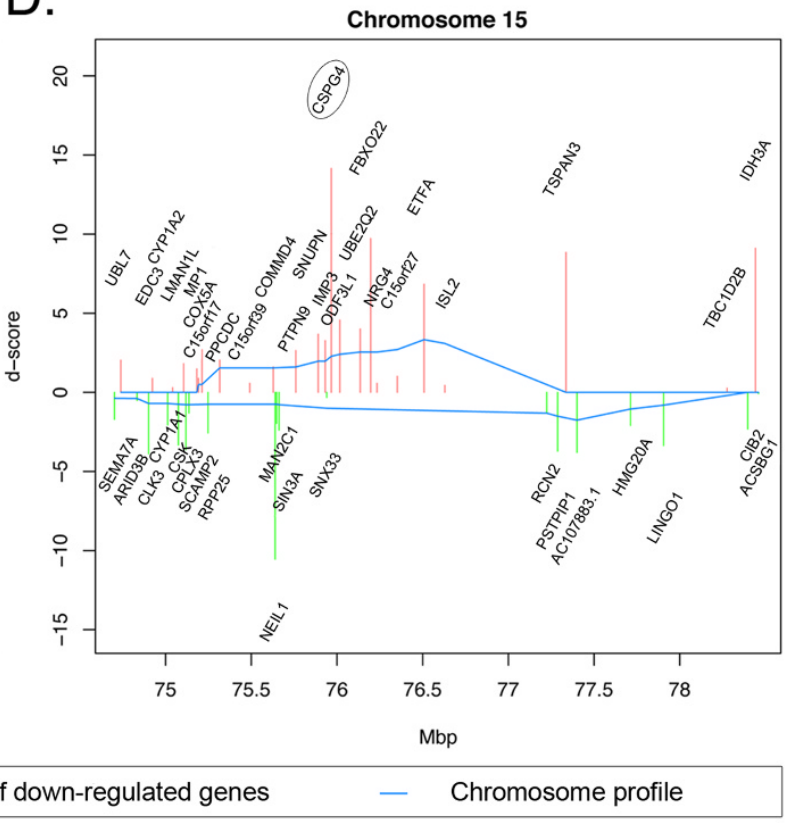

Figure 1 Enlargement of chromosomal regions related to leukaemia phenotype. Details on imbalanced regions calculated by STEPath chromosome mapping. Blue line represents chromosome profile; red and light green bars represent gene statistic values (d-score).

A. Enlargement of the region of chromosome 11 containing the MLL gene (gene highlighted by the circle). B. Enlargement of the region between 20 and $32 \mathrm{Mbp}$ of chromosome 7. This region corresponds to the localization of the HOX gene cluster (cluster highlighted by the circle). C. Enlargement of the region between 51 and $75 \mathrm{Mbp}$ of chromosome 2 corresponding to the MEIS1 windows (gene highlighted by the circle). D. Enlargement of the region of chromosome 15 containing the NG2 gene (gene highlighted by the circle).

ran limma GSEA using the chromosome profile correction. $55.5 \%$ of the common gene sets resulted with lower q-values when the analysis was integrated by the correction for chromosome profile. Furthermore, this correction was able to filter out the glyoxylate cycle, present in bacteria, fungi, yeast and plants (Table 2). This demonstrates that the correction enhances the discovery of disease-related genes, also filtering for apparently not informative pathways (in this case because specific for bacteria, fungi, yeast and plants).

\section{Meta-analysis of LGMDs}

We applied STEPath in a meta-analysis approach involving expression datasets of limb girdle muscular 


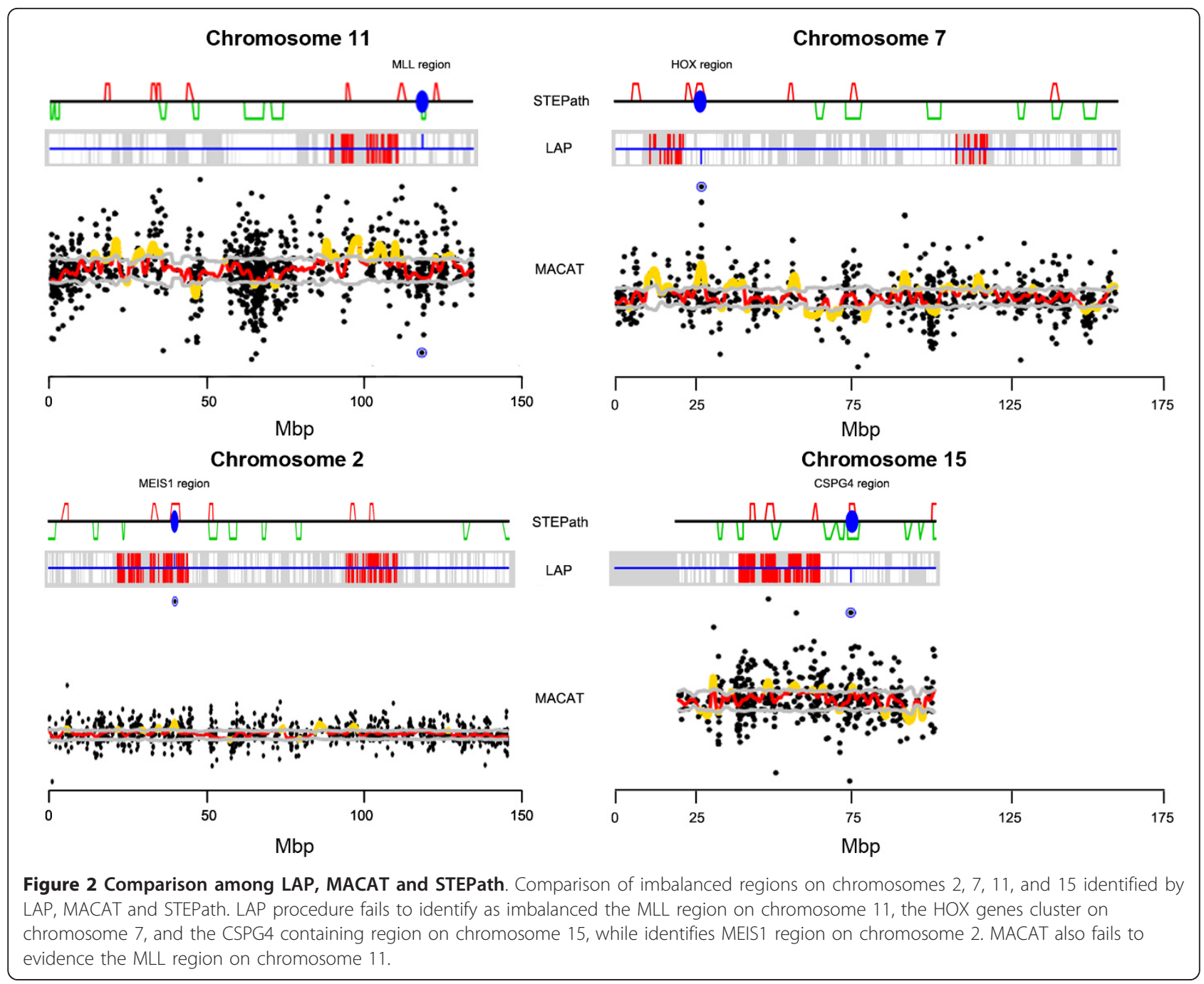

dystrophies type 2A (LGMD2A, calpainopathy), type 2B (LGMD2B, dysferlinopathy) and type 2I (LGMD2I). We built a meta-dataset combining LGMD2A from two distinct datasets. The first was published by Bakay [53] (GEO series ID: GSE3307); the second dataset was published by Sáenz [54] (GEO series ID: GSE11681). Metadataset details are listed in Table 3. Downloaded CEL files were processed using gene-based custom CDF [43]. We retrieved expression for 11,302 distinct genes. Following a visual inspection of the quantiles distribution (boxplot), we excluded 7 control samples from dataset GSE3307 (see Additional file 1; Figure S7). Gene expression data were then globally normalized using the RMA procedure [45].

We applied 4 individual STEPath procedures to CTRLs vs LGMD2A (GSE3307), CTRLs vs LGMD2A (GSE11681), CTRLs vs LGMD2B (GSE3307) and CTRLs vs LGMD2I (GSE3307), where CTRLs are normal muscle controls from healthy donors (Ctrl plus AbNORM in
Table 3). Ensembl features with no corresponding EntrezGene IDs as well as features/genes belonging to the Y chromosome were filtered out. We used STEPath scores to perform gene set meta-analysis (Step 3) (Figure 3).

In general, methods for meta-analysis were based on the union or the intersection of lists of differentially expressed genes derived from multiple studies of the same biological issue. However, these lists have little overlap because of biological and technical variability $[55,56]$, while pathway analysis often generates improved consistency [16]. An explanation for the reduced discrepancies in the results of the microarray data based on biological gene sets analysis, compared with the over imposition of the groups of differentially expressed genes derived from different studies, may be the correlation of differentially expressed genes. In fact, the differences in their relative expression may be so small that the choice of top-ranked genes is highly dependent on 
Table 1 Comparison of GSEA approaches

\begin{tabular}{|c|c|c|}
\hline Rank & STEPath & STEPath - no correction \\
\hline 1 & BioCarta;Erythropoietin mediated neuroprotection through NF-kB & BioCarta;Erythropoietin mediated neuroprotection through NF-kB \\
\hline 2 & SuperArray;Homeobox (HOX) Genes & $\begin{array}{l}\text { BioCarta; Induction of apoptosis through DR3 and DR4/5 Death } \\
\text { Receptors }\end{array}$ \\
\hline 3 & BioCarta;The IGF-1 Receptor and Longevity & $\begin{array}{l}\text { BioCarta; Roles of -arrestin-dependent Recruitment of Src Kinases in } \\
\text { GPCR Signaling }\end{array}$ \\
\hline 4 & $\begin{array}{l}\text { BioCarta;induction of apoptosis through DR3 and DR4/5 Death } \\
\text { Receptors }\end{array}$ & SuperArray;Homeobox (HOX) Genes \\
\hline 5 & $\begin{array}{l}\text { BioCarta;lL12 and Stat4 Dependent Signaling Pathway in Th1 } \\
\text { Development }\end{array}$ & BioCarta;HIV-I Nef negative effector of Fas and TNF \\
\hline 6 & BioCarta;HIV-I Nef negative effector of Fas and TNF & TCA Cycle;Metabolic Process \\
\hline 7 & $\begin{array}{l}\text { BioCarta;Roles of -arrestin-dependent Recruitment of Src Kinases in } \\
\text { GPCR Signaling }\end{array}$ & hsa00310;Lysine degradation \\
\hline 8 & TCA Cycle;Metabolic Process & hsa03018;RNA degradation \\
\hline 9 & hsa00310;Lysine degradation & hsa05014;Amyotrophic lateral sclerosis (ALS) \\
\hline 10 & hsa03018;RNA degradation & SuperArray;Stress/Toxicity PathwayFinder \\
\hline Rank & GSEA - limma & sigPathway \\
\hline 1 & B Cell Receptor Signaling Pathway;Cellular Process & BioCarta;Caspase Cascade in Apoptosis \\
\hline 2 & hsa03018;RNA degradation & KEGG:03050;Proteasome \\
\hline 3 & SuperArray;G-Proteins/Signaling Molecules & KEGG:04130;SNARE interactions in vesicular transport \\
\hline 4 & TNF-alpha/NF-kB Signaling Pathway;Cellular Process & SuperArray;Heat Shock Proteins \\
\hline 5 & hsa00510;N-Glycan biosynthesis & Proteasome Degradation;Physiological Process \\
\hline 6 & hsa04142;Lysosome & KEGG:00380;Tryptophan metabolism \\
\hline 7 & SuperArray;Autophagy & BioCarta;FAS signaling pathway (CD95) \\
\hline 8 & $\begin{array}{l}\text { BioCarta; Erk and PI-3 Kinase Are Necessary for Collagen Binding in } \\
\text { Corneal Epithelia }\end{array}$ & KEGG:04612;Antigen processing and presentation \\
\hline 9 & Translation Factors;Cellular Process & KEGG:03020;RNA polymerase \\
\hline 10 & BioCyc;glyoxylate cycle ॥ & KEGG:00020;Citrate cycle (TCA cycle) \\
\hline
\end{tabular}

Rank comparison of tested GSA for the most 10 up-regulated gene sets. STEPath is the only procedure that was able to identify the activated HOX gene set with a best rank using the corrected expression value based on chromosome profile.

the studies or analysis method from which genes are inferred, as reported in [16]. Moreover there is the possibility that gene sets with no differentially expressed genes (due by the choice of the threshold) will show an aberrant global expression pattern because most of the genes in the set have an even small, but coordinated change (up- or down-regulation) allowing their identification in different studies and increasing their comparability.

Recently, Shen [11] proposed the integrated MetaAnalysis of Pathway Enrichment approach (MAPE_I), combining statistical significance at the gene and pathway level based on a gene-wise and sample-wise permutation test. Our framework is focused on this aspect of meta-analysis, but it introduces the possibility to compare different conditions, highlighting peculiarities of each one.

To produce the main gene set signature (MGS, the union of the pathology signature; see Methods), we chose GSE3307 (LGMD2A, LGMD2B and LGMD2I) as a reference study. The signature was composed of 70 gene sets:
55 Gene Ontology and 15 biological pathways that were used to build the gene set matrix (see Methods).

Cluster analysis of the gene set matrix (derived from the main signature) shows that the two LGMD2As from different datasets are linked, and LGMD2B and LGMD2I segregate separately (Figure $4 \mathrm{~B}$; for data matrix see Additional file 3).

We then performed unsupervised cluster analysis [57] on gene expression data. This analysis highlighted two main branches that separate GSE3307 and GSE11681 datasets (Figure 4A). This demonstrates that background noise and the presence of a batch effect is strong enough to overcome disease-specific signals at gene expression levels (Figure 4A). Moreover, cluster analysis failed to separate pathologies (see Additional file 1; Figure S8) using significant differentially expressed genes only (significant genes identified by SAM with False Discovery Rate $=0$ ).

Our approach overcomes the main limitations of gene expression meta-analysis and demonstrates that it is useful to reveal gene set signatures that discriminate 
Table 2 GSEA approach results running limma GSEA with and without chromosome profile correction

\begin{tabular}{lllll}
\hline Rank & GSEA - limma & q-value & GSEA - limma - corrected & q-value \\
\hline 1 & B Cell Receptor Signaling Pathway;Cellular Process & $1.62 \mathrm{E}-05$ & B Cell Receptor Signaling Pathway;Cellular Process & $2.68 \mathrm{E}-05$ \\
\hline 2 & hsa03018;RNA degradation & $1.44 \mathrm{E}-03$ & SuperArray;G-Proteins/Signaling Molecules & $1.33 \mathrm{E}-03$ \\
\hline 3 & SuperArray;G-Proteins/Signaling Molecules & $1.53 \mathrm{E}-03$ & hsa03018;RNA degradation & $2.31 \mathrm{E}-03$ \\
\hline 4 & TNF-alpha/NF-kB Signaling Pathway;Cellular Process & $3.79 \mathrm{E}-03$ & TNF-alpha/NF-kB Signaling Pathway;Cellular Process & $2.93 \mathrm{E}-03$ \\
\hline 5 & hsa00510;N-Glycan biosynthesis & $4.20 \mathrm{E}-03$ & Translation Factors;Cellular Process & $5.30 \mathrm{E}-03$ \\
\hline 6 & hsa04142;Lysosome & $8.78 \mathrm{E}-03$ & hsa00510;N-Glycan biosynthesis & $5.40 \mathrm{E}-03$ \\
\hline 7 & SuperArray;Autophagy & $8.95 \mathrm{E}-03$ & hsa04142;Lysosome & $6.40 \mathrm{E}-03$ \\
\hline 8 & $\begin{array}{l}\text { BioCarta;Erk and Pl-3 Kinase Are Necessary for } \\
\text { Collagen Binding in Corneal Epithelia }\end{array}$ & $9.36 \mathrm{E}-03$ & SuperArray;Autophagy & $8.21 \mathrm{E}-03$ \\
\hline 9 & Translation Factors;Cellular Process & $9.38 \mathrm{E}-03$ & hsa05110;Vibrio cholerae infection & $9.42 \mathrm{E}-03$ \\
\hline 10 & BioCyc;glyoxylate cycle II & $1.06 \mathrm{E}-02$ & BioCarta;Erk and Pl-3 Kinase Are Necessary for & $9.42 \mathrm{E}-03$ \\
& & & Collagen Binding in Corneal Epithelia & \\
\hline
\end{tabular}

Limma GSEA algorithm was run using the chromosome profile correction. Significance of the differentially expressed gene sets increases in comparison with results obtained without introducing chromosome profile correction, suggesting that it targets disease-related genes.

different pathologies. In this way, we can evaluate the main signature discrimination/association power, projecting it into the second study (Figure 3, point 3).

\section{Meta-analysis of Skeletal Muscular diseases}

We extended the analysis performed for LGMDs including more variability with different skeletal muscular diseases (all samples reported in the Table 3).

Raw expression files (CEL files) were downloaded from the GEO database [7] (GEO series IDs GSE3307 and GSE11681) and processed using a gene-based custom CDF, as previously discussed. Normalized gene expression for 11,302 genes was used in the STEPath analysis. Extended datasets were used to evaluate if increased variability affects the meta-analysis procedure.
We extracted signatures from all skeletal muscle diseases in the dataset GSE3307 to build the MGS used in the cluster analysis. Cluster analysis showed that increased variability of initial samples did not affect clustering results since LGMDs still clustered together; different datasets of LGMD2A were still in close proximity (Figure 4C; for matrix, see Additional file 4).

We compared STEPath meta-analysis results with a meta-analysis approach based on different GSA scores. Similarly for STEPath, we built a MGS matrix using scores derived by both sigPathway and GSEA (as implemented in limma). Clustering results of the MGS matrix from both sigPathway and GSEA failed to co-segregate the two LGMD2A datasets and the entire group of LGMDs (Figure 4D and 4E).

Table 3 Details of muscle disease dataset

\begin{tabular}{llclll}
\hline Disease & $\begin{array}{l}\text { Number of } \\
\text { samples }\end{array}$ & $\begin{array}{l}\text { Case } \\
\text { study }\end{array}$ & Series ID & Platform & Description \\
\hline LGMD2A & 10 & L/S & GSE3307 & HGU133A & MUSCULAR DYSTROPHY, LIMB-GIRDLE, TYPE 2A (calpainopathy) \\
\hline nLGMD2A & 10 & L/S & GSE11681 & HGU133A & MUSCULAR DYSTROPHY, LIMB-GIRDLE, TYPE 2A (calpainopathy) \\
\hline LGMD2B & 10 & L/S & GSE3307 & HGU133A & $\begin{array}{l}\text { MUSCULAR DYSTROPHY, LIMB-GIRDLE, TYPE 2B (Dysferlinopathy, Miyoshi distal } \\
\text { myopathy) }\end{array}$ \\
\hline LGMD21 & 7 & L/S & GSE3307 & HGU133A & MUSCULAR DYSTROPHY, LIMB-GIRDLE, TYPE 2I \\
\hline BMD & 5 & S & GSE3307 & HGU133A & MUSCULAR DYSTROPHY, BECKER TYPE \\
\hline DMD & 10 & S & GSE3307 & HGU133A & MUSCULAR DYSTROPHY, PSEUDOHYPERTROPHIC PROGRESSIVE, DUCHENNE TYPE \\
\hline FSHD & 14 & S & GSE3307 & HGU133A & MUSCULAR DYSTROPHY, FACIOSCAPULOHUMERAL \\
\hline AQM & 5 & S & GSE3307 & HGU133A & ACUTE QUADRIPLEGIC MYOPATHY \\
\hline SPG4 & 4 & $\mathrm{~S}$ & GSE3307 & HGU133A & SPASTIC PARAPLEGIA 4, AUTOSOMAL DOMINANT \\
\hline ALS & 9 & S & GSE3307 & HGU133A & AMYOTROPHIC LATERAL SCLEROSIS 1 \\
\hline X_EDMD & 4 & S & GSE3307 & HGU133A & EMERY-DREIFUSS MUSCULAR DYSTROPHY, 1 (X-linked) \\
\hline AD_EDMD & 4 & $S$ & GSE3307 & HGU133A & EMERY-DREIFUSS MUSCULAR DYSTROPHY, AUTOSOMAL DOMINANT \\
\hline AbNORM & 11 & L/S & GSE3307 & HGU133A & NORMAL \\
\hline Ctrl & 10 & L/S & GSE11681 & HGU133A & NORMAL \\
\hline
\end{tabular}




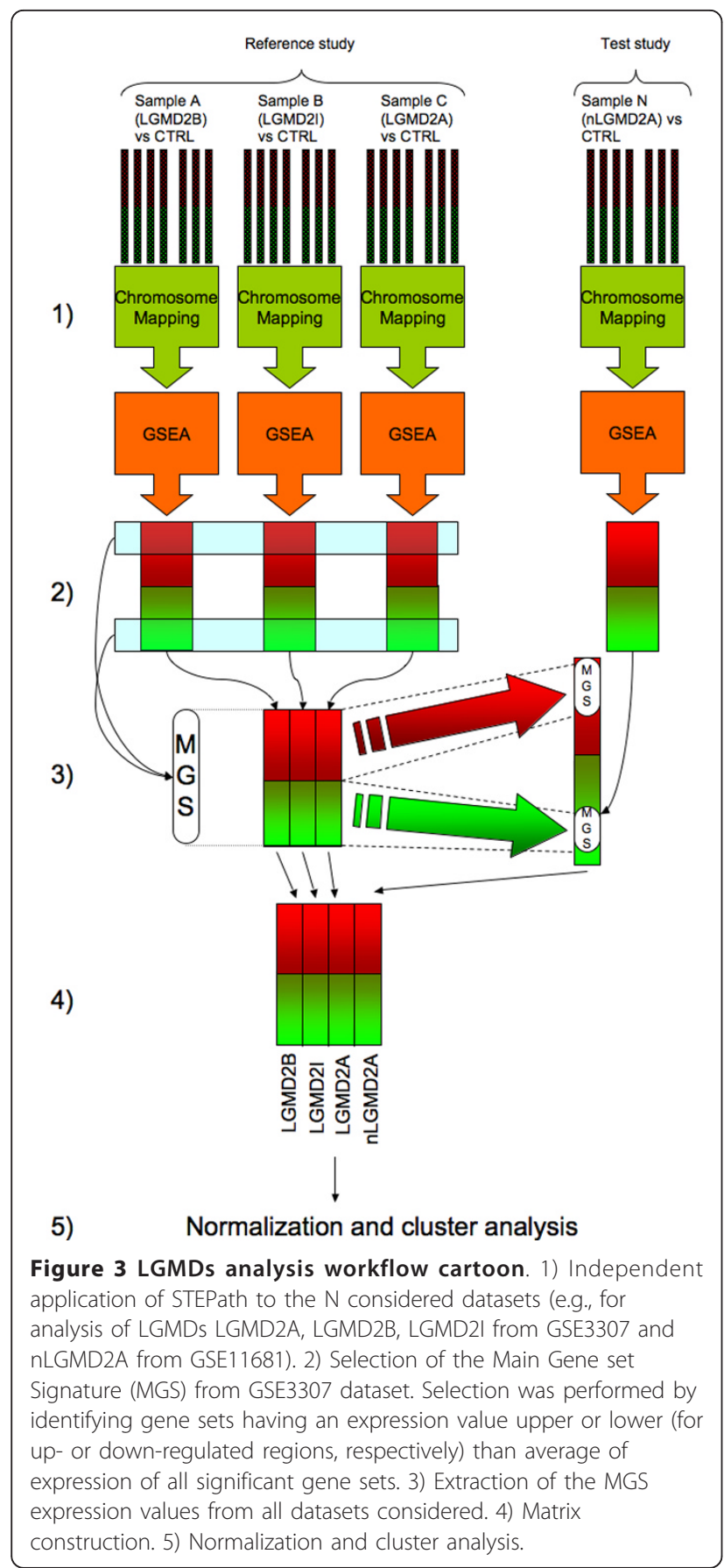

Gene set clusters were analysed focusing on both shared and peculiar pathology responses. Down-regulated gene sets show several clusters with the same expression level. These gene sets mainly refer to aerobic respiration, the production of ATP and mitochondria (Figure 5). These results are in agreement with many microarray studies on skeletal muscle dystrophies [58] and a previous meta-analysis work that we performed to detect muscle atrophy signatures [59]. In many skeletal muscle pathologies, the rate of degradation of contractile proteins becomes greater than the rate of replacement, causing atrophy and modifying the balance requested for the maintenance of skeletal muscle mass. Ubiquitination function involved in protein degradation and gene sets for oxidative stress and mitochondrial function appear to be up-regulated, yet they are not discriminative among the pathologies. Gene sets involved in oxidoreductase activity (GO_MF: 0016641), scavenger receptor activity (GO_MF: 0005044) and regulation of amino acids (GO_BP: 0045764 and GO_BP: 0001934) are some examples (Figure 5). Recently, Kramerova [60] postulated that LGMD2A and other dystrophies (Duchenne Muscular Distrophy and Becker Muscular Dystrophy) are characterized by energy deficit and increased oxidative stress. We highlighted the activation of gene sets involved in antioxidant activity like GO_MF: 0016681, GO_MF: 0016679 and GO_MF:0016641 that referred to oxidoreductase activity.

Dysferlinopathy (LGMD2B) was characterized as dystrophies where dysferlin-deficient cells show abnormalities in vesicular trafficking [61]. LGMD2B also presents muscle inflammation with muscular monocytes and macrophages that show an increased phagocytic activity [62]. Efficient phagocytic activity depends on the presence of the coat protein complex type I (COPI) [63], a complex that plays an essential role in the trafficking of membrane vesicles. Our procedure reveals this relation between vesicle trafficking impairment and enhanced phagocytosis in LGMD2B as is demonstrated by a marked up-regulation of GO terms that refer to COPIcoated vesicles (GO_CC: 0030137, GO_CC: 0030663 and GO_CC: 0030126) (Figure 5).

To search for discriminative gene sets among LGMD2A (Calpain 3; CAPN3 is the causative gene) and the other skeletal muscular diseases, we performed a template matching search [64]. In Figure 6 are reported hierarchical clusters of gene sets identified with $\mathrm{p}$-value $\leq 0.05$.

Recently, Beckman and Spencer [65] proposed that CAPN3 is involved in sarcomeric protein turnover and in the maintenance of sarcomere integrity. Collagen (GO_CC: 0005581), collagen binding (GO_MF: 0005518) and fibrillar collagen (GO_CC: 0005583) gene sets, involved in the maintenance of mechanical stability of muscle structure, sarcomere (GO_CC: 0030017), striated muscle thick filament (GO_CC: 0005863), A band (GO_CC: 0031672) and contractile fiber (GO_CC: 0043292) involved in the contraction process, appear to be up-regulated in LGMD2A (Figure 6). Gene sets previously described are also altered in LGMD2B patients that present mutations in the dysferlin gene (DYSF), which interacts with CAPN3. A secondary reduction of CAPN3 that can affect sarcomere structure stability in LGMD2B patients has also been demonstrated [66]. 


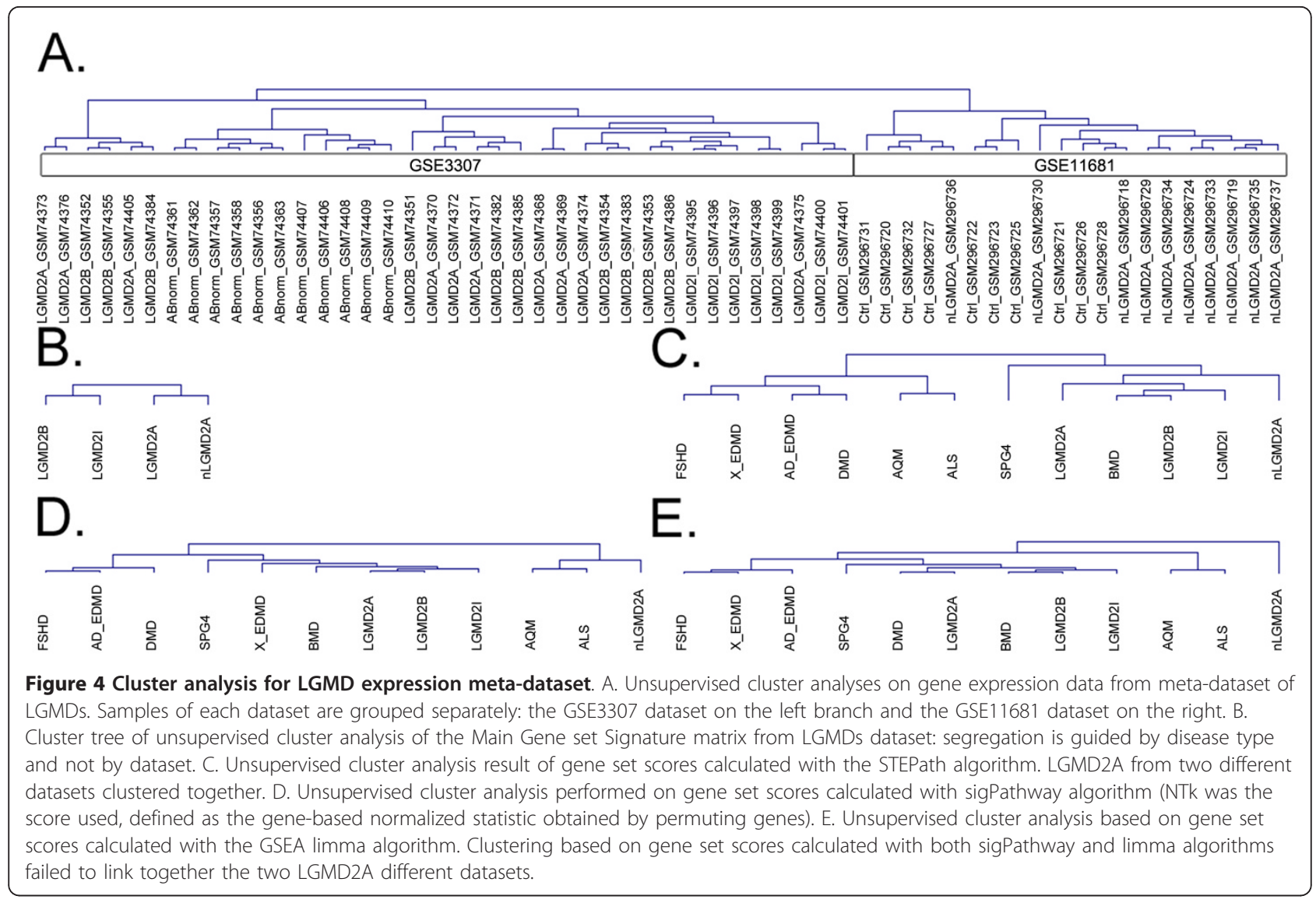

\section{Implementation}

All functions to perform STEPath analysis are written in R (http://www.r-project.org/, version 2.10.1), and they are freely available as $\mathrm{R}$ package at http://gefu.cribi. unipd.it/papers/stepath under the AGPL3 licence. The implementation depends on bioconductor [67] version 2.5 (for affy R package [68]) and samr R package (http:// CRAN.R-project.org/package $=$ samr).

The present implementation is microarray platformindependent and potentially could be applied to any kind of gene-phenotype association score (SAM t-test, t-test).

\section{Conclusions}

The algorithm we developed allows the analysis of gene expression data by integrating supplementary biological information to identify gene co-expression along the chromosomes and to perform a gene set analysis. The integration was initially tested on a leukaemia dataset, highlighting interesting imbalanced regions containing genes involved in ALL pathology: 1) MLL region on chromosome $11 ; 2$ ) the region containing HOX gene cluster on chromosome 7; and 3) the region containing MEIS1 on chromosome 2 . These regions are specifically enhanced by the STEPath algorithm and not by LAP or MACAT.
The second integration was tested on gene expression datasets both from leukaemia and skeletal muscle diseases evidencing the importance of integration of the chromosome profiles in the gene set analysis. Integrating two biological aspects in the STEPath algorithm (locus transcription that spills over into its physical neighbour loci and co-regulation of gene sets), we have demonstrated that STEPath produces gene set expression scores that are suitable to directly compare different diseases and studies to perform meta-analyses.

We applied STEPath and the meta-analysis approach to limb girdle muscular dystrophies (LGMDs), highlighting the co-segregation of two different studies of LGMD2A patients, and to a meta-dataset for inflammatory myopathies composed of both Affymetrix arrays and unpublished custom oligo arrays. Results of the study of inflammatory myopathies will be discussed in a separate paper.

\section{Methods}

\section{STEPath algorithm}

Step 1: The process to identify differentially expressed regions can be divided into four parts: I) computation of a per-gene statistic to measure differences in gene expression between two groups under investigation or 


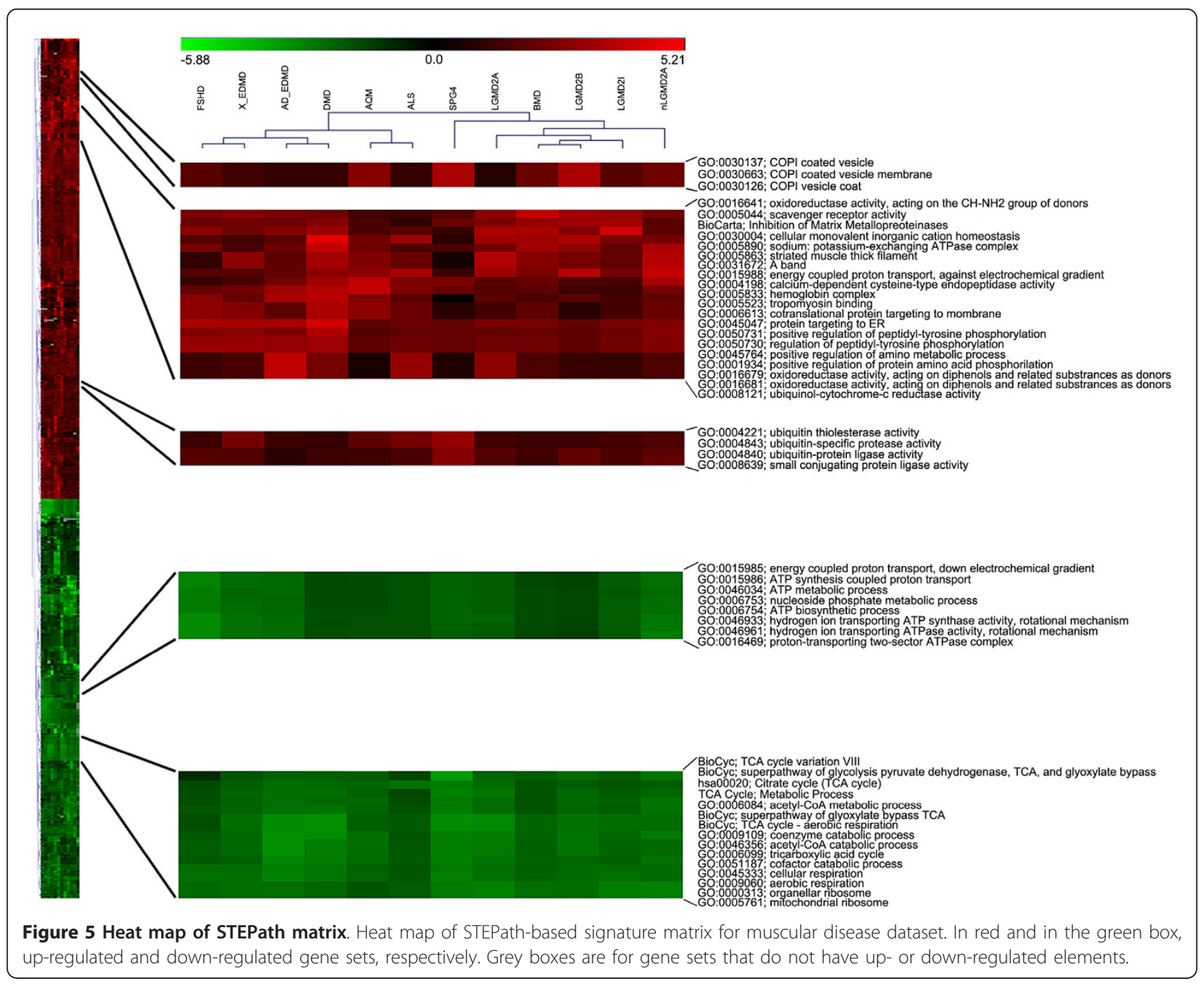

in one group, if microarray experiments were performed in a competitive hybridization way, II) correction of the statistics based on the expression level of neighbourhood loci, III) identification of statistically differentially expressed regions by a permutational approach and IV) building of per-chromosome profile.

I) In this study, we used the SAM t-statistic to measure the association of genes to the phenotype of the two conditions. All SAM analyses were computed using two-class unpaired comparisons between a disease state versus a reference condition based on 100 permutations via the samr $R$ package [69].

II) This step computes the local index of global activation (Eup) or inhibition (Edown) for every gene $i$ considering the neighbour genes and the local gene density.

For any given gene $i$, rup $_{i}$ is the region centred in the transcription start site of the gene $i$ (TSS $i$ ) that covers $\mathrm{n}$ $=2$ up-regulated genes upstream TSS $i$ and $n=2$ upregulated genes downstream TSSi. Given rup $i$, we can summarize the local gene expression contribution for up-regulated genes inside the region $\left(S_{i}^{\prime \text { up }}\right)$, as described in equation 1 :

$$
S_{i}^{\prime u p}=\frac{\sum_{u \in U} S_{u}}{|U|},
$$

where $U=\left\{u \in \operatorname{rup}_{i} \mid S_{u} \geq 0\right\}$, and $|U|$ denotes the cardinality of the set $U$.

We can also define the local gene density of up-regulated genes $\left(\rho_{\text {up }}\right)$ as reported in equation 2 :

$$
\rho_{i}^{u p}=\frac{|U|}{\text { length }\left(\text { rup }_{i}\right)} .
$$

$\operatorname{Eup}_{i}$ is then calculated as define by equation (3):

$$
E u p_{i}=\frac{S_{i}^{u p} \bullet \rho_{i}^{u p}}{S_{c}^{u p} \bullet \rho_{c}^{u p}},
$$




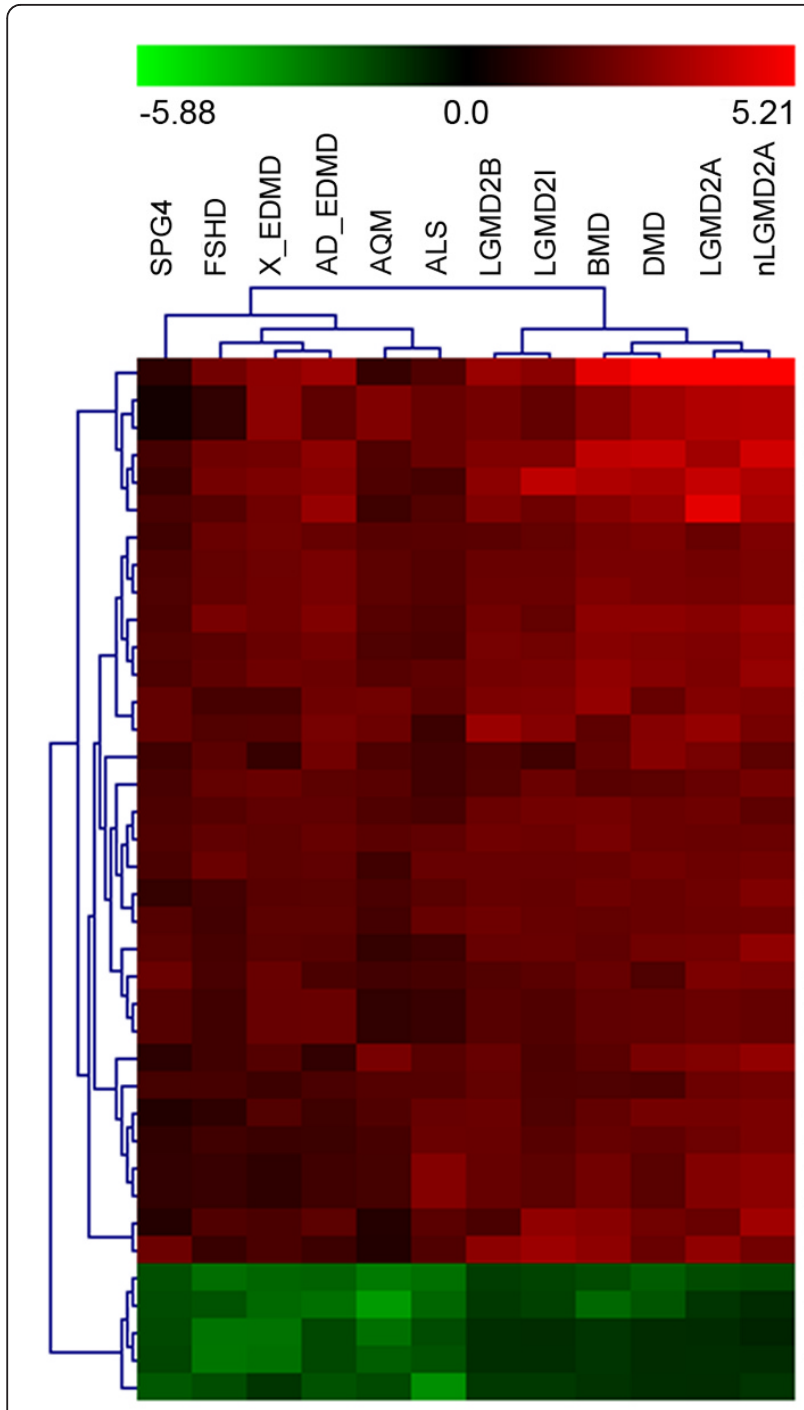

GO:0005583; fibrillar collagen

GO:0005863; striated muscle thick filament

GO:0031672; A band

GO:0005581; collagen

GO:0005518; collagen binding

BioCarta; Small Leucine-rich Proteoglycan (SLRP) molecules

SuperArray; Osteogenesis

GO:0031012; extracellular matrix

GO:0005578; proteinaceous extracellular matrix

GO:0005201; extracellular matrix structural constituent

hsa04512; ECM-receptor interaction

GO:0006817; phosphate transport

GO:0005788; endoplasmic reticulum lumen

BioCarta; IL 4 signaling pathway

BioCarta; The IGF-1 Receptor and Longevity

Prostaglandin Synthesis and Regulation; Metabolic Process

SuperArray; Extracellular Matrix 9 Adhesion Molecules

hsa04510; Focal adhesion

GO:0051258; protein polymerization

GO:00164559; myosin complex

hsa05416; Viral myocarditis

Inflammatory Response Pathway; Physiological Process

GO:0004177; aminopeptidase activity

GO:0043062; extracellular structure organization and biogenesis GO:0030198; extracellular matrix organization and biogenesis

GO:0006941; striated muscle contraction

hsa04260; Cardiac muscle contraction

GO:0030017; sarcomere

GO:0043292; contractile fiber

GO:0016460; myosin II complex

GO:0005859; muscle myosin complex

GO:0005021; vascular endothelial growth factorreceptor activity

GO:0051238; sequestering of metal ion

hsa04260; Cardiac muscle contraction

GO:0008543; fibroblast growth factor receptor signaling pathway

hsa03010; Ribosome

Cytoplasmic Ribosomal Proteins; Molecular Function

BioCarta; mCalpain and friends in Cell motility

Figure 6 Heat map of PTM analysis. Heat map of PTM analysis searching for gene sets with marked up-regulation in LGMD2A pathologies with respect to other gene sets.

where $S^{\text {sup }}{ }_{c}$ is the mean of summarization values for all designed rup in the chromosome $c$ and $\rho^{u p}{ }_{c}$ is a perchromosome estimation of the global up-regulated gene density. We defined per-chromosome global up-regulated gene density as the mean of all local densities for every up-regulated gene.

The ratio between $S^{\prime}{ }_{i}{ }_{i}$ and $S^{\prime}{ }_{c}{ }_{c}$ is meant to rescale regional expression compared to the average situation in the chromosome $c$, while the ratio between $\rho^{u p}{ }_{i}$ and $\rho^{u p}{ }_{c}$ is meant to favour regions presenting genes more densely distributed than the mean local densities of the chromosome.

In parallel, for any gene $i$, we defined $r d o w n_{i}$ and the summarization value $E d o w n_{i}$ as described for up-regulated genes. In this case, $U$ is defined as follows:

$$
U=\left\{u \in r \text { down }_{i} \mid S_{u}<0\right\} .
$$

III) We adopted a permutational approach to identify significantly different $\operatorname{Eup}_{i}$ and Edown ${ }_{i}$. In particular, SAM statistics were randomly shuffled over gene positions. We applied procedure II) and III) to $B=100$ of these permutations to compute $E^{\prime} u p_{i, b}$ and $E^{\prime} d o w n_{i, b}$ null distributions, where $1 \leq b \leq B$ (null hypothesis $H^{0}$ : the region is not differentially expressed). We computed $\mathrm{p}$-values for every window centred in gene $i$ as the probability that $E^{\prime} u p_{i}$ or $E^{\prime} d o w n_{i}$ exceed respectively the observed $\operatorname{Eup}_{i}$ and $E_{\text {down }}$ over $B$ permutations. We then corrected $\mathrm{p}$-values for multiple testing error using the Benjamini Hochberg FDR control (preprocessCore $r$ package [70]). 
IV) The final step was performed to produce a perchromosome profile by scanning each chromosome gene by gene using a window of fixed length. For window size, we used the reference lengths for up- and down-windows defined as the average length of all rup $i$ and $r d o w n_{i}$ for a given chromosome $c$. We used these dimensions because they are in accordance with the clustering scale dimensions found in mammalian genomes by Firneisz [71] and Farr [72] (see Additional file 2 , Table S5). We count significant (q-value $<=0.05$ ) up- or down-regulated window expression values present in the fixed windows that slide gene by gene. The up and down profile was built respectively as the fraction of significant Eup or Edown present in the sliding windows.

\section{Statistics smoothing based on chromosome profile function}

SAM statistic $S$ was corrected according to equation 4:

$$
S S_{i}=S_{i}+\left(S_{i} \cdot p f_{i}\right)
$$

where $S S_{i}$ is the smoothed statistic value for gene $i$, and $p f_{i}$ is the profile value in the chromosome region identified by the gene $i$ (see step IV of the previous paragraph).

Step 2: the GSA module can be divided into two parts: 1) computation of per-gene set scores and 2) identification of significant gene sets.

1) We implemented the measurement of gene set scores as the mean of the corrected SAM statistics, SS. In particular, we performed the GSA computing up- and down-regulated gene contributions separately. Let the indices $k, k=1, \ldots, K$ denotes the $k^{\text {th }}$ gene set, and $i, i=$ $1, . ., I$ denotes the $i^{t h}$ gene. We defined an incidence matrix $M$ with dimensions $K \times I$, where $M_{k, i}=1$ denotes the presence of gene $i$ in the $k^{t h}$ gene set, and $M_{k, i}=0$ denotes the absence of gene $i$ in the $k^{\text {th }}$ gene set. We computed a gene set up-regulation value $\operatorname{Gup}_{k}$ for the $k^{\text {th }}$ gene set as the mean of $S S_{i\lfloor k} \geq 0$ and similarly for downregulated values $\left(G d o w n_{k}\right)$.

2) To assess the significance of gene sets, we adopted a gene-based permutational scheme to compute null distributions of $S S: S S^{\prime}$. We applied $B=100$ permutations on $S$, and for each permutation $b$, we smoothed $S_{b}{ }_{b}$ to compute $S S_{b}^{\prime}$ (null hypothesis $H^{0}$ : the gene set shows the same pattern of association with the phenotype compared to the rest of genes). We applied the procedure described in 1) to compute $\mathrm{Gup}_{b}{ }_{b}$ and Gdown' ${ }_{b}$ null distributions for each gene set. P-values were then calculated for Gup $p_{k}$ and Gdown $n_{k}$ independently as the probability that Gup' ${ }_{k}$ or Gdown' ${ }_{k}$ exceed the observed gene set score $G u p_{k}$ or $G$ down $n_{k}$ over $B=100$ permutations. P-values were finally corrected according to Benjamini Hochberg FDR control.
Step 3: step 3 compares different pathologies and different studies. The starting points are Gup and Gdown summarization values of the gene sets from step 2 .

A reference study was defined as the one with greater variability. From each pathology in the reference study, a gene set signature was extracted. It was defined as the significant gene sets (q-value $<=0.05$ ) with either Gup or Gdown exceeding the mean score of significant gene sets.

We called the union of the pathology signatures main gene set signature (MGS). Using the MGS, corresponding summarization values from all the pathologies in all studies (from the reference study and from the validating one) were extracted and a matrix (MGS matrix) was produced, where columns were the different pathologies, and rows were summarization values for every gene set on the signature. To make comparable values of each gene set signature among different pathologies, quantile normalization was applied. The normalized matrix provides a direct comparison of gene set activation and inhibition across pathologies and studies. We adopted this strategy because signatures identified in one study should be identified in independent studies for the same pathology; related pathologies from different studies should cluster together.

\section{Custom CDF}

We developed a gene-based custom Chip Definition File (CDF) by re-mapping probes of Affymetrix HGU133plus 2 and HGU133A chips on the ENSEMBL gene database (ver 56). Gene-based custom CDFs were generated as follows: i) matching of ensemble gene sequences with all probes present in a given gene chip (HGU133plus2, HGU133A); ii) filtering out of non-specific probes (probes that match more than one gene sequence); iii) grouping of remaining probes in meta-probe sets with at least 4 members; iv) discarding all probes not belonging to any meta-probe set defined in point iii) [43]. None of the identified genes share TSS in both of the designed CDFs. We adopted this strategy because gene sets are defined as groups of genes and not as groups of transcripts that could derive from alternative TSS of the same gene.

\section{Gene sets}

We compiled a collection of gene sets using various public databases. We used gene sets from Gene Ontology (GO) (6,466 gene sets derived from Biological Process, Molecular Function and Cellular Component), 204 KEGG pathways, 161 ByoCyc pathways, 102 Superarray pathways and 111 wiki pathways. Only gene sets with members in the CDF were used in the analysis.

\section{Normalization}

Multichip normalization was performed using RMA as implemented in the affy bioconductor package. 
The gene set expression matrix was normalized by quantile normalization as implemented in $\mathrm{R}$ package preprocessCore.

\section{Cluster and Template Matching Analysis}

All cluster analyses were performed using the Euclidean distance with complete linkage method. We used the Hierarchical Cluster Analysis (HCL) implemented in the TMeV suite (version 3.1) [73].

PTM analysis was performed using the PTM function implemented in the $\mathrm{TMeV}$ suite and setting $\mathrm{p}$-value threshold at 0.05 .

\section{$R$ packages}

Limma GSEA was performed using the limma bioconductor R package.

SigPathway analysis was performed using the sigpathway bioconductor $\mathrm{R}$ package.

MACAT analysis was performed using the macat bioconductor R package.

\section{Additional material}

Additional file 1: Additional figures. Word document containing supplementary figures. The figures are provided one per page with a short description.

Additional file 2: Additional tables. Excel spreadsheet file containing supplementary tables. External link to NCBI and Gene Card databases are provided for genes explored in the chromosome regions described in the text.

Additional file 3: LGMDs dataset data matrix. Text file containing data matrix for LGMD dataset gene set meta-analysis.

Additional file 4: Muscle disease dataset data matrix. Text file containing data matrix for muscular disease dataset gene set meta-analysis.

\section{List of abbreviations}

LAP: Locally Adaptive statistical Procedure; MACAT: MicroArray Chromosome Analysis Tool; AML: acute myeloid Leukaemia; ALL: acute lymphoblastic leukaemia; GSA: Gene Set Analysis; SAM: Statistical Analysis of Microarray; KEGG: Kyoto Encyclopedia of Genes and Genomes; GEO: Gene Expression Omnibus; RMA: Robust Multiarray Averaging; PAM: Predicted Analysis of Microarray; PCOT2: Principal Coordinates and Hotelling's T²; SAFE: Significance Analysis of Function and Expression, LGMDs: Limb Girdle Muscular Dystrophies; CTRL: Control; MAPE, Meta-Analysis of Pathway Enrichment; MGS: Main Gene set Signature; ATP: Adenosine triphosphate; GO_BP: Gene Ontology Biological Process; GO_MF: Gene Ontology Molecular Function; GO_CC: Gene Ontology Cellular Component; AGPL3: GNU Affero General Public License; FDR: False Discovery Rate; CDF: Chip Definition File; HCL: Hierarchical Cluster Analysis; TMeV: TIGR MultiExperiment Viewer; PTM: Pavlidis Template Matching.

\section{Acknowledgements}

This work was supported by a grant from Association Française contre les Myopathies (AFM) call 2008 to G. L.

\section{Author details}

'CRIBI Biotechnology Centre, University of Padova, via U. Bassi 58/B, 35121 Padova, Italy. ${ }^{2}$ Department of Biology, University of Padova, via U. Bassi 58/B, 35121 Padova, Italy. ${ }^{3}$ Department of Statistical Science, University of Padova, via C. Battisti 241, 35121 Padova, Italy.

\section{Authors' contributions}

PM implemented the STEPath algorithm, performed all of the statistical and bioinformatic analyses. DR, GS and CR participated in the design of the study, revised the manuscript and participated in the investigation of the significant gene sets and interpretation of the results. GL and SC conceived and supervised the study, wrote the manuscript, coordinated the work and the interpretation of the results. All authors read and approved the final version of the manuscript declaring that they have no potential conflicts of interests.

\section{Competing interests}

The authors declare that they have no competing interests.

Received: 8 October 2010 Accepted: 11 April 2011

Published: 11 April 2011

\section{References}

1. Brennan DJ, Kelly C, Rexhepaj E, Dervan PA, Duffy MJ, Gallagher WM: Contribution of DNA and tissue microarray technology to the identification and validation of biomarkers and personalised medicine in breast cancer. Cancer Genomics Proteomics 2007, 4(3):121-134.

2. Chen Y, Miller C, Mosher R, Zhao X, Deeds J, Morrissey M, Bryant B, Yang D, Meyer $R$, Cronin $F$, et al: Identification of cervical cancer markers by cDNA and tissue microarrays. Cancer Res 2003, 63(8):1927-1935.

3. Malinowski DP: Multiple biomarkers in molecular oncology. II. Molecular diagnostics applications in breast cancer management. Expert Rev Mol Diagn 2007, 7(3):269-280.

4. Malinowski DP: Multiple biomarkers in molecular oncology. I. Molecular diagnostics applications in cervical cancer detection. Expert Rev Mol Diagn 2007, 7(2):117-131.

5. Olson JA Jr: Application of microarray profiling to clinical trials in cancer. Surgery 2004, 136(3):519-523.

6. Sun Y, Goodison S, Li J, Liu L, Farmerie W: Improved breast cancer prognosis through the combination of clinical and genetic markers. Bioinformatics 2007, 23(1):30-37.

7. Barrett T, Troup DB, Wilhite SE, Ledoux P, Rudnev D, Evangelista C, Kim IF, Soboleva A, Tomashevsky M, Marshall KA, et al: NCBI GEO: archive for high-throughput functional genomic data. Nucleic Acids Res 2009, , 37 Database: D885-890.

8. Brazma A, Hingamp P, Quackenbush J, Sherlock G, Spellman P, Stoeckert C, Aach J, Ansorge W, Ball CA, Causton HC, et al: Minimum information about a microarray experiment (MIAME)-toward standards for microarray data. Nat Genet 2001, 29(4):365-371

9. Rocca-Serra P, Brazma A, Parkinson H, Sarkans U, Shojatalab M, Contrino S, Vilo J, Abeygunawardena N, Mukherjee G, Holloway E, et al: ArrayExpress: a public database of gene expression data at EBI. C R Biol 2003, 326(1011):1075-1078

10. Goeman JJ, Buhlmann P: Analyzing gene expression data in terms of gene sets: methodological issues. Bioinformatics 2007, 23(8):980-987.

11. Shen K, Tseng GC: Meta-analysis for pathway enrichment analysis when combining multiple genomic studies. Bioinformatics 26(10):1316-1323.

12. Normand SL: Meta-analysis: formulating, evaluating, combining, and reporting. Stat Med 1999, 18(3):321-359.

13. Rhodes DR, Barrette TR, Rubin MA, Ghosh D, Chinnaiyan AM: Meta-analysis of microarrays: interstudy validation of gene expression profiles reveals pathway dysregulation in prostate cancer. Cancer Res 2002, 62(15):4427-4433.

14. Parmigiani G, Garrett-Mayer ES, Anbazhagan R, Gabrielson E: A cross-study comparison of gene expression studies for the molecular classification of lung cancer. Clin Cancer Res 2004, 10(9):2922-2927.

15. Park WD, Stegall MD: A meta-analysis of kidney microarray datasets: investigation of cytokine gene detection and correlation with rt-PCR and detection thresholds. BMC Genomics 2007, 8:88.

16. Manoli T, Gretz N, Grone HJ, Kenzelmann M, Eils R, Brors B: Group testing for pathway analysis improves comparability of different microarray datasets. Bioinformatics 2006, 22(20):2500-2506.

17. Callegaro A, Basso D, Bicciato S: A locally adaptive statistical procedure (LAP) to identify differentially expressed chromosomal regions. Bioinformatics 2006, 22(21):2658-2666.

18. Toedling J, Schmeier S, Heinig M, Georgi B, Roepcke S: MACAT-microarray chromosome analysis tool. Bioinformatics 2005, 21(9):2112-2113. 
19. Turkheimer FE, Roncaroli F, Hennuy B, Herens C, Nguyen M, Martin D, Evrard A, Bours V, Boniver J, Deprez M: Chromosomal patterns of gene expression from microarray data: methodology, validation and clinical relevance in gliomas. BMC Bioinformatics 2006, 7:526.

20. Barry WT, Nobel AB, Wright FA: Significance analysis of functional categories in gene expression studies: a structured permutation approach. Bioinformatics 2005, 21(9):1943-1949.

21. Goeman JJ, van de Geer SA, de Kort F, van Houwelingen HC: A global test for groups of genes: testing association with a clinical outcome. Bioinformatics 2004, 20(1):93-99.

22. Subramanian A, Tamayo P, Mootha VK, Mukherjee S, Ebert BL, Gillette MA, Paulovich A, Pomeroy SL, Golub TR, Lander ES, et al: Gene set enrichment analysis: a knowledge-based approach for interpreting genome-wide expression profiles. Proc Natl Acad Sci USA 2005, 102(43):15545-15550.

23. Tian L, Greenberg SA, Kong SW, Altschuler J, Kohane IS, Park PJ: Discovering statistically significant pathways in expression profiling studies. Proc Natl Acad Sci USA 2005, 102(38):13544-13549.

24. Levin AM, Ghosh D, Cho KR, Kardia SL: A model-based scan statistic for identifying extreme chromosomal regions of gene expression in human tumors. Bioinformatics 2005, 21(12):2867-2874.

25. Anderson AN, Roncaroli F, Hodges A, Deprez M, Turkheimer FE: Chromosomal profiles of gene expression in Huntington's disease. Brain 2008, 131(Pt 2):381-388.

26. Efron and Tibshirani: On testing the significance of sets of genes. Ann App/ Stat 2007, 1:107-129.

27. Szabo PM, Tamasi V, Molnar V, Andrasfalvy M, Tombol Z, Farkas R, Kovesdi K, Patocs A, Toth M, Szalai C, et al: Meta-analysis of adrenocortical tumour genomics data: novel pathogenic pathways revealed. Oncogene 29(21):3163-3172.

28. Sinha B, Bhattacharya D, Sinha DK, Talwar S, Maharana S, Gupta S, Shivashankar GV: Dynamic organization of chromatin assembly and transcription factories in living cells. Methods Cell Biol 98:57-78.

29. van Steensel B, Dekker J: Genomics tools for unraveling chromosome architecture. Nat Biotechnol 28(10):1089-1095.

30. Meaburn KJ, Misteli T: Cell biology: chromosome territories. Nature 2007, 445(7126):379-781

31. Ebisuya M, Yamamoto $T$, Nakajima M, Nishida E: Ripples from neighbouring transcription. Nat Cell Biol 2008, 10(9):1106-1113.

32. Tusher VG, Tibshirani R, Chu G: Significance analysis of microarrays applied to the ionizing radiation response. Proc Natl Acad Sci USA 2001, 98(9):5116-5121.

33. Ashburner M, Ball CA, Blake JA, Botstein D, Butler H, Cherry JM, Davis AP, Dolinski K, Dwight SS, Eppig JT, et al: Gene ontology: tool for the unification of biology. The Gene Ontology Consortium. Nat Genet 2000, 25(1):25-29.

34. Kanehisa M, Goto S: KEGG: kyoto encyclopedia of genes and genomes. Nucleic Acids Res 2000, 28(1):27-30.

35. Kanehisa M, Goto S, Furumichi M, Tanabe M, Hirakawa M: KEGG for representation and analysis of molecular networks involving diseases and drugs. Nucleic Acids Res, 38 Database: D355-360.

36. Kanehisa M, Goto S, Hattori M, Aoki-Kinoshita KF, Itoh M, Kawashima S, Katayama T, Araki M, Hirakawa M: From genomics to chemical genomics: new developments in KEGG. Nucleic Acids Res 2006, , 34 Database: D354-357.

37. Karp PD, Ouzounis CA, Moore-Kochlacs C, Goldovsky L, Kaipa P, Ahren D, Tsoka S, Darzentas N, Kunin V, Lopez-Bigas N: Expansion of the BioCyc collection of pathway/genome databases to 160 genomes. Nucleic Acids Res 2005, 33(19):6083-6089.

38. BioCarta. [http://www.biocarta.com].

39. SuperArray. [http://www.sabiosciences.com/pathwaycentral.php].

40. WikiPathways. [http://www.wikipathways.org].

41. Song S, Black MA: Microarray-based gene set analysis: a comparison of current methods. BMC Bioinformatics 2008, 9:502.

42. Benjamini $Y$, Hochberg $Y$ : Controlling the false discovery rate: a practical and powerful approach to multiple testing. J R Statist Soc B 1995, 289-300.

43. Sales G, Coppe A, Bicciato S, Bortoluzzi S, Romualdi C: Impact of probe annotation on the integration of miRNA-mRNA expression profiles for miRNA target detection. Nucleic Acids Res 38(7):e97.

44. Dai M, Wang P, Boyd AD, Kostov G, Athey B, Jones EG, Bunney WE, Myers RM, Speed TP, Akil H, et al: Evolving gene/transcript definitions significantly alter the interpretation of GeneChip data. Nucleic Acids Res 2005, 33(20):e175.

45. Irizarry RA, Hobbs B, Collin F, Beazer-Barclay YD, Antonellis KJ, Scherf U, Speed TP: Exploration, normalization, and summaries of high density oligonucleotide array probe level data. Biostatistics 2003, 4(2):249-264.

46. Krivtsov AV, Armstrong SA: MLL translocations, histone modifications and leukaemia stem-cell development. Nat Rev Cancer 2007, 7(11):823-833

47. Zangrando A, Dell'orto MC, Te Kronnie G, Basso G: MLL rearrangements in pediatric acute lymphoblastic and myeloblastic leukemias: MLL specific and lineage specific signatures. BMC Med Genomics 2009, 2:36.

48. Hilden JM, Smith FO, Frestedt JL, McGlennen R, Howells WB, Sorensen PH, Arthur DC, Woods WG, Buckley J, Bernstein ID, et al: MLL gene rearrangement, cytogenetic 11q23 abnormalities, and expression of the NG2 molecule in infant acute myeloid leukemia. Blood 1997, 89(10):3801-3805.

49. Tibshirani R, Hastie T, Narasimhan B, Chu G: Diagnosis of multiple cancer types by shrunken centroids of gene expression. Proc Natl Acad Sci USA 2002, 99(10):6567-6572.

50. Kong SW, Pu WT, Park PJ: A multivariate approach for integrating genome-wide expression data and biological knowledge. Bioinformatics 2006, 22(19):2373-2380.

51. Ferrando AA, Armstrong SA, Neuberg DS, Sallan SE, Silverman LB, Korsmeyer SJ, Look AT: Gene expression signatures in MLL-rearranged T-lineage and B-precursor acute leukemias: dominance of HOX dysregulation. Blood 2003, 102(1):262-268.

52. Argiropoulos B, Humphries RK: Hox genes in hematopoiesis and leukemogenesis. Oncogene 2007, 26(47):6766-6776.

53. Bakay M, Wang Z, Melcon G, Schiltz L, Xuan J, Zhao P, Sartorelli V, Seo J, Pegoraro E, Angelini $C$, et al: Nuclear envelope dystrophies show a transcriptional fingerprint suggesting disruption of Rb-MyoD pathways in muscle regeneration. Brain 2006, 129(Pt 4):996-1013.

54. Saenz A, Azpitarte M, Armananzas R, Leturcq F, Alzualde A, Inza I, GarciaBragado F, De la Herran G, Corcuera J, Cabello A, et al: Gene expression profiling in limb-girdle muscular dystrophy 2A. PLoS One 2008, 3(11): e3750.

55. Ein-Dor L, Kela I, Getz G, Givol D, Domany E: Outcome signature genes in breast cancer: is there a unique set? Bioinformatics 2005, 21(2):171-178.

56. Tan PK, Downey TJ, Spitznagel EL Jr, Xu P, Fu D, Dimitrov DS, Lempicki RA, Raaka BM, Cam MC: Evaluation of gene expression measurements from commercial microarray platforms. Nucleic Acids Res 2003, 31(19):5676-5684

57. Eisen MB, Spellman PT, Brown PO, Botstein D: Cluster analysis and display of genome-wide expression patterns. Proc Natl Acad Sci USA 1998, 95(25):14863-14868.

58. Campanaro S, Romualdi C, Fanin M, Celegato B, Pacchioni B, Trevisan S, Laveder P, De Pitta C, Pegoraro E, Hayashi YK, et al: Gene expression profiling in dysferlinopathies using a dedicated muscle microarray. Hum Mol Genet 2002, 11(26):3283-3298.

59. Calura E, Cagnin S, Raffaello A, Laveder P, Lanfranchi G, Romualdi C: Metaanalysis of expression signatures of muscle atrophy: gene interaction networks in early and late stages. BMC Genomics 2008, 9:630.

60. Kramerova I, Kudryashova E, Wu B, Germain S, Vandenborne K, Romain N, Haller RG, Verity MA, Spencer MJ: Mitochondrial abnormalities, energy deficit and oxidative stress are features of calpain 3 deficiency in skeletal muscle. Hum Mol Genet 2009, 18(17):3194-3205.

61. Kesari A, Fukuda M, Knoblach S, Bashir R, Nader GA, Rao D, Nagaraju K, Hoffman EP: Dysferlin deficiency shows compensatory induction of Rab27A/SIp2a that may contribute to inflammatory onset. Am J Pathol 2008, 173(5):1476-1487.

62. Nagaraju K, Rawat R, Veszelovszky E, Thapliyal R, Kesari A, Sparks S, Raben N, Plotz P, Hoffman EP: Dysferlin deficiency enhances monocyte phagocytosis: a model for the inflammatory onset of limb-girdle muscular dystrophy 2B. Am J Pathol 2008, 172(3):774-785.

63. Hackam DJ, Botelho RJ, Sjolin C, Rotstein OD, Robinson JM, Schreiber AD, Grinstein S: Indirect role for COPI in the completion of FCgamma receptor-mediated phagocytosis. J Biol Chem 2001, 276(21):18200-18208.

64. Pavlidis $P$, Noble WS: Analysis of strain and regional variation in gene expression in mouse brain. Genome Biol 2001, 2(10):RESEARCH0042.

65. Beckmann JS, Spencer M: Calpain 3, the "gatekeeper" of proper sarcomere assembly, turnover and maintenance. Neuromuscul Disord 2008, 18(12):913-921. 
66. Huang $Y$, Verheesen $P$, Roussis A, Frankhuizen W, Ginjaar I, Haldane F, Laval S, Anderson LV, Verrips T, Frants RR, et al: Protein studies in dysferlinopathy patients using llama-derived antibody fragments selected by phage display. Eur J Hum Genet 2005, 13(6):721-730.

67. Gentleman RC, Carey VJ, Bates DM, Bolstad B, Dettling M, Dudoit S, Ellis B, Gautier L, Ge Y, Gentry J, et al: Bioconductor: open software development for computational biology and bioinformatics. Genome Biol 2004, 5(10): R80.

68. Gautier L, Cope L, Bolstad BM, Irizarry RA: affy-analysis of Affymetrix GeneChip data at the probe level. Bioinformatics 2004, 20(3):307-315

69. Team RDC: R: A Language and Environment for Statistical Computing. 2009

70. Bolstad BM: preprocessCore: A collection of pre-processing functions.

71. Firneisz G, Zehavi I, Vermes C, Hanyecz A, Frieman JA, Glant TT: Identification and quantification of disease-related gene clusters. Bioinformatics 2003, 19(14):1781-1786.

72. Farr CJ, Goodfellow PN: Hidden messages in genetic maps. Science 1992, 258(5079):49.

73. Saeed Al, Sharov V, White J, Li J, Liang W, Bhagabati N, Braisted J, Klapa M, Currier $T$, Thiagarajan $M$, et al: TM4: a free, open-source system for microarray data management and analysis. Biotechniques 2003, 34(2):374-378.

doi:10.1186/1471-2105-12-92

Cite this article as: Martini et al.: Statistical Test of Expression Pattern (STEPath): a new strategy to integrate gene expression data with genomic information in individual and meta-analysis studies. $B M C$ Bioinformatics 2011 12.92.

\section{Submit your next manuscript to BioMed Central} and take full advantage of:

- Convenient online submission

- Thorough peer review

- No space constraints or color figure charges

- Immediate publication on acceptance

- Inclusion in PubMed, CAS, Scopus and Google Scholar

- Research which is freely available for redistribution

Submit your manuscript at www.biomedcentral.com/submit 